\title{
Sintomatologia Depressiva, Atenção Sustentada e Desempenho Escolar em Estudantes do Ensino Médio
}

\author{
Depressão, atenção e desempenho
}

\author{
Makilim Nunes Baptista \\ Ricardo Franco de Lima \\ Alessandra Gotuzo Seabra Capovilla \\ Liana Lins Melo
}

\section{Resumo}

A depressão inclui diversos sintomas cognitivos, tais como problemas de atenção que, por sua vez, podem prejudicar o desempenho escolar. De modo a verificar as relações entre essas variáveis, foram obtidas as notas escolares e aplicados o Teste de Atenção Concentrada e o Inventário de Depressão de Beck a 62 estudantes do ensino médio, de ambos os sexos, com idades entre 15 e 24 anos. Destes, 33,8\% apresentaram sintomas depressivos, distribuídos entre os níveis leve e grave, sendo que as mulheres apresentaram maior severidade de sintomas que os homens. A severidade de sintomatologia depressiva mostrou-se negativamente correlacionada tanto com o desempenho escolar quanto com a qualidade da atenção. A comparação entre grupos com e sem sintomas de depressão revelaram que o primeiro apresentou desempenho escolar pior e déficit da atenção. Tais resultados corroboram a hipótese de relação entre depressão, atenção e desempenho escolar em alunos do Ensino Médio.

Palavras-chave: Depressão; Atenção; Desempenho escolar

\section{Depressive symptomatology, sustained attention and academic performance in secondary school students}

\begin{abstract}
Depression includes many cognitive symptoms, such as attention issues that can harm school performance. In order to verify the relations between these variables, school grades were obtained together with the application of the Sustained Attention Test and Beck Depression Inventory among 62 high school students, from both genders, aging from 15 to 24 years old. From these, 33.8\% presented depression symptoms distributed in mild and severe levels, while women present higher levels of symptoms than men do. The severity of depressive symptoms showed itself negatively correlated with school performance and the attention quality. Comparison between groups with and without depressive symptoms revealed that the first one presented lower school performance and attention quality. Such results corroborate the hypothesis of relation between depression, attention and school performance in high school students.
\end{abstract}

Keyword: Depression; Attention; Academic achievement

\section{Síntomas de depresión, atención sostenida y desempeño escolar en estudiantes de educación secundaria.}

\section{Resumen}

\footnotetext{
La depresión incluye síntomas cognitivos diversos tales como problemas de atención, que a su vez pueden perjudicar el desempeño escolar. Como una forma de verificar las relaciones entre esas variables han sido obtenidas las notas escolares y aplicados el Test de Atenção Concentrada y el Inventario de Depresión de Beck en 62 estudiantes de educación secundaria, de ambos sexos, con edades entre 15 y 24 años. Del total de participantes, 33,8\% poseían síntomas de depresión distribuidos entre los niveles leve y grave, siendo que las mujeres poseían una mayor seriedad de síntomas que los hombres. La seriedad de síntomas de depresión se ha mostrado negativamente correlacionada con el desempeño escolar y con la calidad de la atención. La comparación entre los grupos con y sin síntomas de depresión ha mostrado que el primer grupo ha presentado un peor desempeño escolar y un déficit de la atención. Esos resultados confirman la hipótesis sobre la relación entre depresión, atención y desempeño escolar en alumnos de educación secundaria.

Palabras clave: Depresión; Atención; Desempeño escolar
} 


\section{Introdução}

O interesse pelo estudo da depressão em crianças e adolescentes é recente, pois até aproximadamente 1960 acreditava-se que o quadro depressivo nessas faixas etárias era raro ou inexistente. Há evidências recentes de que o quadro de Depressão Maior, por exemplo, pode ocorrer na adolescência por volta dos 13 aos 19 anos, sendo que o primeiro episódio geralmente dura de cinco a nove meses (Bahls, 2002a).

Considerando a natureza multifatorial da depressão, isto é, o fato de não haver um único fator responsável pelo seu desencadeamento, desenvolvimento e manutenção, pode-se dizer que existem fatores de risco que aumentam a vulnerabilidade e predisposição no aparecimento da sintomatologia (Baptista \& Assumpção Junior, 1999). Entre os fatores de risco estão os ambientais tais como o histórico de vida com ausência de suporte familiar e social, a percepção da falta desse suporte, o número acumulado de mudanças no curso de vida (de endereço, desemprego dos pais, mudanças na estrutura familiar), e as dificuldades psicossociais crônicas e as perdas, que podem favorecer o desencadeamento de crises na adolescência. Além desses, fatores como doenças, uso de medicamentos e abuso de substâncias psicoativas podem gerar sintomas depressivos secundários (Graeff \& Brandão, 1993; Kaltiala-Heino, Rimpelä, Rantanen \& Laippala, 200I).

Outro fator de vulnerabilidade à depressão é o estresse. Nota-se, geralmente, a ocorrência de estresse severo nos três meses que precedem o aparecimento de uma crise ou episódio depressivo (Graeff \& Brandão, 1993). Do ponto de vista neurológico, evidências sugerem que a exposição ao estresse crônico e o conseqüente aumento dos níveis de cortisol são responsáveis por uma atrofia hipocampal e pelos déficits cognitivos presentes nos quadros depressivos (Joca, Padovan \& Guimarães, 2003).

Os dados da literatura sobre a prevalência da depressão na adolescência possuem alta variabilidade. Em um estudo epidemiológico realizado por Bahls (2002b), em uma escola pública com 463 estudantes do Ensino Fundamental e Médio, com faixa etária entre 10 e 17 anos, verificou-se que $20,3 \%$ dos participantes apresentaram sintomas depressivos significativos, conforme avaliado pelo Inventário de Depressão Infantil (CDI). Houve diferença significativa entre os sintomas em relação ao gênero, com maior prevalência em meninas (72,3 \%) e provável pico de sintomas entre 12 e 15 anos. O estudo de Barbosa, Dias, Gaião e Lourenzo (1996) mostrou uma incidência semelhante. Foram avaliados 807 alunos de escolas da Paraíba, com idades entre 7 e 17 anos, também por intermédio do CDI, sendo que $22 \%$ deles apresentaram sintomatologia depressiva.

Porém, a prevalência de sintomatologia depressiva pode variar de forma brusca, provavelmente devido ao uso de diferentes instrumentos e metodologias. Por exemplo, em um estudo realizado por Baptista e Golfeto (2000), com I 35 estudantes de 07 a 14 anos, também usando o $\mathrm{CDI}$, foi encontrada prevalência baixa de sintomas depressivos ( $1,48 \%)$, que correspondeu a dois indivíduos da amostra total, ambos do sexo feminino. Já ao avaliar 270 estudantes universitários, Goreinstein e Andrade (1998) encontraram, aproximadamente, $13 \%$ com sintomatologia expressiva de depressão.

Em outro estudo, Baptista e Oliveira (2004) avaliaram 154 adolescentes de uma escola pública, também usando o CDI. Destes, 5,6\% apresentaram sintomatologia depressiva significativa. A associação entre depressão e alguns fatores foi ainda observada, tais como a ocorrência de eventos estressantes nos últimos seis meses, alguém na família ter apresentado depressão, ter um dos pais falecidos ou estar tomando medicação psiquiátrica. Conforme discutido por Baptista e Campos (2000), a adolescência tem como característica a variação do humor, o que pode justificar tais discrepâncias na incidência de depressão, nessa faixa de desenvolvimento. Tais variações, porém, podem ser temporárias e não há, necessariamente, a manutenção dos sintomas no decorrer do tempo.

Em relação ao gênero, estudos têm indicado maior prevalência do transtorno entre as mulheres. Em uma pesquisa que avaliou a depressão em adolescentes com o Inventário de Depressão de Beck (BDI), Kaltiala-Heino e cols. (200I) encontraram uma porcentagem maior de meninas com sintomas depressivos nas três classificações, leve, moderada e grave (II,2\%, 9,0\% 
e 2,1\%, respectivamente), quando comparadas aos meninos (5,9\%, 4,7 \% e I,5 \%). A prevalência maior de sintomas em indivíduos do gênero feminino também foi encontrada em outros estudos (Goreinstein \& Andrade, 1998; Kaltiala-Heino \& cols., 200I; Bahls, 2002b; Cruvinel, 2003), explicada pelas diferenças hormonais e psicossociais entre os gêneros (Baptista \& Assumpção Junior, 1999; Baptista, Baptista \& Oliveira, 2004; Kornstein, 1997; Soares, Almeida-Filho, Botega, Coutinho \& Mari, 1994).

Kornstein (1997) pontua, em seu artigo de revisão bibliográfica, que mulheres desenvolvem episódios de depressão mais longos e crônicos, são mais sensíveis aos eventos de vida como desencadeantes de episódios depressivos, e são mais suscetíveis a tais episódios em função das alterações hormonais constantes. As mulheres, além de apresentarem uma incidência aproximadamente duas vezes maior de prevalência de sintomas depressivos que os homens, também apresentam diferenças em relação ao curso da doença e às respostas aos tratamentos medicamentoso e psicológico.

Os sintomas depressivos são variados e podem ser classificados em afetivos, neurovegetativos, ideativos, cognitivos. Estão relacionados à autovaloração, à volição e à psicomotricidade (Dalgalarrondo, 2000). Dentre os sintomas cognitivos da depressão, o rebaixamento na atenção, nas funções executivas e na memória são as alterações neuropsicológicas que correspondem mais diretamente ao critério cognitivo "prejuízo na capacidade de pensar ou concentrar", definido pelo DSM-IV-TR para o diagnóstico de Depressão Maior (American Psychiatric Association, 2000).

A atenção é uma função neuropsicológica básica que está subjacente a todos os processos cognitivos. Pode ser definida como a capacidade do indivíduo selecionar e focalizar seus processos mentais em algum aspecto do ambiente interno ou externo, respondendo predominantemente aos estímulos que Ihe são significativos e inibindo respostas aos demais estímulos (Brandão, 1995; Engelhardt, Rozenthal \& Laks, 1996).

Existem diferentes tipos de atenção em função do tipo de processamento envolvido, incluindo atenção seletiva, atenção sustentada, atenção alternada e atenção dividida (Sternberg, 2000). Serão enfocadas, no presente trabalho, a atenção seletiva e a sustentada. Atenção seletiva refere-se à capacidade de emitir respostas a um estímulo específico desconsiderando aqueles não relevantes. Atenção sustentada representa a capacidade do indivíduo em mantê-la focalizada em uma seqüência de estímulos, por um período de tempo, para conseguir desempenhar determinada tarefa, sendo caracterizada por uma habilidade em detectar estímulos (nível de vigilância) e por uma diminuição no desempenho ao longo do tempo. Cada aspecto da atenção relaciona-se a regiões e estruturas encefálicas específicas que atuam como um sistema integrado (Sarter, Givens \& Bruno, 200I).

Os estudos com Tomografia por Emissão de Pósitrons (TEP) realizados com humanos sugerem um sistema fronto-parietal no hemisfério direito para a atenção sustentada. Especificamente no transtorno depressivo, os estudos com TEP scan mostram hipometabolismo no córtex pré-frontal dorsal no hemisfério direito e no cingulado anterior, regiões conhecidas por subsidiarem o processamento da atenção em indivíduos normais (Liotti \& Mayberg, 200I). De acordo com Steingard (2000), o lobo frontal também está envolvido na patogênese da depressão em crianças e adolescentes.

Do ponto de vista da psicologia cognitiva, segundo Eysenck e Keane (1994), os indivíduos depressivos apresentam déficit na seletividade atencional com a percepção do meio como uma fonte desinteressante de informações e uma redução da capacidade atencional, pois seus recursos de processamento estão voltados para preocupações consigo mesmo. Esses indivíduos apresentam um foco atencional disperso e reduzido, sendo pouco atentos para mudanças ocorridas no ambiente.

Diversos fatores podem influenciar o desempenho em tarefas de atenção. Por exemplo, Silva e Leite (2000) conduziram um estudo com o teste de Stroop e verificaram que características específicas do teste (no caso, necessidade de realização em dois minutos e indicação de erros por um sinal) podem atuar como fator ansiogênico e influenciar o desempenho. Naquele estudo, os autores informaram aos participantes que 
eles deveriam executar a tarefa o mais rápido possível, porém que, por se tratar de um teste de atenção, não adiantaria preencher a folha totalmente, mas com muitos erros. Apesar das instruções, a resolução parece ter sido influenciada pela expectativa do preenchimento completo do teste e pela autocobrança quanto ao bom desempenho.

Assim, a consideração do tempo como um fator de dificuldade pelos participantes e a interferência da rapidez na qualidade podem indicar uma possível relação entre as variáveis tempo e qualidade da atenção. Especificamente em relação à depressão, essa interferência pode ser interpretada como uma dificuldade dos indivíduos com maior prevalência de sintomas depressivos em selecionar estímulos e manter o foco atencional na tarefa desempenhada, dadas as evidências de baixo escore de qualidade da atenção (Silva \& Leite, 2000).

Resultados semelhantes têm sido encontrados em diagnóstico de depressão (Rozenthal, Laks \& Engelhardt, 2004). Indivíduos depressivos tendem a apresentar número maior de omissões no Continuous Performance Test e déficits no processamento temporal quando comparados com grupo de sujeitos sem depressão (Sévigny, Everett \& Grondin, 2003). Quando comparados aos indivíduos esquizofrênicos, os depressivos tendem a apresentar redução da vigilância (concentração) em teste de atenção seletiva e sustentada (Egeland \& cols., 2003).

Também têm sido relatados déficits na atenção direcionada e na memória de trabalho (Williams \& cols., 2000). Em testes de atenção dividida, os indivíduos depressivos tendem a apresentar aumento significativo do tempo de reação, sugerindo falha na distribuição dos recursos mentais e na regulação da atenção (Thomas \& Rousseaux, 1998).

Outros estudos têm sido conduzidos considerando que os transtornos afetivos e ansiosos estão associados a diferentes padrões cognitivos de processamento de estímulos no que se refere ao conteúdo e preferência de processamento, possibilitando uma maior caracterização do déficit. Em uma pesquisa realizada por Mathews, Ridgeway e Williamson (1996) verificou-se que os indivíduos depressivos foram seletivamente mais atentos para palavras socialmente ameaçadoras enquanto que os ansiosos tenderam a selecionar palavras de ameaça física. Esses estudos fornecem evidências de que a orientação da atenção pode estar relacionada para estímulos que são emocionalmente relevantes para a pessoa.

É interessante notar, porém, que há alguns estudos com resultados contraditórios a respeito da presença do déficit atencional em indivíduos depressivos. Por exemplo, dentre 16 estudos revisados por Ottowitz, Dougherty e Savage (2002) que avaliavam a atenção, menos da metade (44\%) indicaram a presença de um déficit nos indivíduos depressivos. Em uma pesquisa realizada por Lautenbacher, Spernal e Christiankrieg (2002), foi encontrado prejuízo em indivíduos depressivos quando comparados a indivíduos com transtorno de pânico apenas em paradigmas de atenção dividida, mas não na atenção seletiva.

Alguns fatores podem elucidar essas contradições, tais como as diferenças na administração dos testes, descrições, versões dos testes utilizadas e controle de variáveis. Além disso, diversas variáveis interferem no estudo das relações entre depressão e funções neuropsicológicas, pois ambos são fenômenos multifacetados. A depressão possui subtipos, sintomatologia ampla e pode afetar alguns determinados componentes da atenção, mas não outros. Neste caso, esses déficits seriam identificados apenas por meio de testes designados para a avaliação do componente específico que está prejudicado (Ottowitz \& cols., 2002). Porém, independentemente das disparidades nos resultados das pesquisas, Thomas e cols. (1998) sugerem que as medidas neuropsicológicas podem ter utilidade clínica na detecção precoce de sintomas depressivos, ou mesmo na detecção de subtipos de sintomas residuais de um episódio agudo que podem prejudicar $\circ$ funcionamento cognitivo ou fornecer sinal de recaída.

Tais déficits cognitivos presentes na sintomatologia depressiva podem repercutir em outros aspectos da vida do indivíduo (Ottowitz, Dougherty \& Savage, 2002). De fato, a depressão em adolescentes geralmente é acompanhada por prejuízos no desempenho escolar e nas relações sociais, e aumenta o risco do abuso de substâncias, o que pode ser prevenido por intervenções precoces (Weller \& Weller, 2000). 
Em uma revisão da literatura envolvendo depressão, Barbosa (2004) verificou que a depressão em estudantes aumenta os afastamentos da escola, atrasos, problemas de comportamento e conduta, isolamento social, risco de suicídio, abuso de substâncias, redução do interesse, motivação e na participação em atividades, redução dos processos cognitivos, da atenção e concentração e do desempenho escolar geral, incluindo leitura, redação e aritmética. Em uma pesquisa realizada por Pérez e Urquijo (200I) com 888 adolescentes, o aumento dos níveis de depressão dos estudantes correlacionase à diminuição do desempenho escolar em línguas e matemática. Dell'Aglio e Hutz (2004) também verificaram uma correlação negativa entre sintomas depressivos e desempenho escolar em alunos.

Tais dados tornam-se ainda mais importantes diante da realidade escolar brasileira, em que há um grande número de alunos com desempenho rebaixado. Podese constatar no Exame Nacional de Ensino Médio (ENEM) de 2005, considerando apenas as questões objetivas, que $60,2 \%$ participantes tiveram desempenhos na faixa de insuficiente a regular, $34,9 \%$ entre regular e bom, e apenas $4,9 \%$ de bom a excelente (Instituto Nacional de Estudos e Pesquisas Educacionais Anísio Teixeira - INEP, 2005). É urgente, portanto, expandir a compreensão das possíveis variáveis que influenciam o desempenho escolar, de forma a promover procedimentos interventivos eficazes. Nesse contexto, o estudo das relações entre depressão, atenção e desempenho escolar torna-se fundamental.

Corroborando a relação entre tais fatores, Cruvinel (2003) verificou que os sintomas depressivos na infância e adolescência interferem negativamente no uso de estratégias de aprendizagem e no rendimento escolar, principalmente em matemática. Segundo a autora, os processos cognitivos desses indivíduos são disfuncionais, pois apresentam um estilo negativo de avaliarem a si mesmos, ao mundo e ao futuro. Além disso, tendem a selecionar e privilegiar os eventos negativos de suas vidas e é provável que, diante de uma situação de fracasso escolar, nutram pensamentos exagerados de autodesvalorização, desesperança e pessimismo. Desse modo, seus processos cognitivos podem influenciar a motivação, o desempenho e a aprendizagem, o que ressalta a importância de estudar a depressão e suas possíveis relações com a escolarização formal de indivíduos nos diferentes níveis de escolaridade.

Aliada a tais dificuldades, a falta de informações de pais e professores sobre a depressão na adolescência pode contribuir para o aumento das dificuldades enfrentadas pelo estudante. Um olhar mais atento pode permitir a identificação precoce e o encaminhamento adequado (Barbosa, 2004).

Dadas as relações entre atenção, sintomatologia depressiva e desempenho escolar indicadas na literatura, o presente estudo teve como objetivo verificar possíveis associações entre essas variáveis em estudantes do Ensino Médio. É importante ressaltar, porém, que, apesar da relação entre processos cognitivos, afetivos e desempenho escolar, há outros fatores envolvidos no processo de aprendizagem, tais como os métodos de ensino do professor e a orientação pedagógica da escola, por exemplo. Não se deve incorrer no erro de atribuir o desempenho escolar inadequado exclusivamente a falhas atencionais ou a transtornos de humor, visto que o estudante é um ser biopsicosocial e diferentes fatores atuam, conjuntamente, no processo de aprendizagem formal (Terigi \& Baquero, 1997).

\section{Método}

\section{Participantes}

Participaram do estudo 62 estudantes cursando o $1^{\circ}$ ano (5\%), $2^{\circ}$ ano (53\%) e $3^{\circ}$ ano (42\%) do Ensino Médio de uma escola do interior paulista. A idade média geral foi de 17,I, com desvio-padrão de I,4 anos, sendo 26 participantes do sexo masculino (42 $\%)$ e 36 do sexo feminino (58\%).

\section{Instrumentos}

Nesta pesquisa foram utilizados dois instrumentos, o Teste de Atenção Concentrada Toulousse-Pieron e - Inventário de Depressão de Beck, além de uma ficha para obtenção dos dados pessoais e as notas escolares dos participantes. 
Teste de Atenção Concentrada Toulousse Pieron (Fator P) da Bateria Fatorial CEPA (CEPA, 200I)

O teste tem a duração de cinco minutos e fornece quatro escores: a) Escore bruto e percentil em rapidez, que corresponde ao número de linhas que os participantes conseguem percorrer durante a administração do teste. Quanto maior o percentil, mais rápido a resolução do teste; b) Escore bruto e percentil em qualidade, calculado pela soma de erros (itens assinalados erradamente) e omissões (itens que $\circ$ participante deveria assinalar, porém não assinalou). Portanto, quanto maior o escore, maior é o número de erros e/ou omissões (pior qualidade). Na conversão para o percentil, quanto menos erros, maior o percentil;

\section{Inventário de Depressão de Beck ("Beck}

Depression Inventory" - BDI) (Cunha, 200I)

O inventário foi utilizado para avaliar a sintomatologia depressiva. É composto por 21 itens que se referem a sintomas e atitudes, devendo o sujeito escolher qual a intensidade que corresponde ao seu estado atual, numa escala que varia de 0 a 3 para cada item;

\section{Ficha para obtenção dos dados pessoais}

Foi incluída uma escala subjetiva de alerta, com duas perguntas sobre a percepção do próprio indivíduo sobre a sua atenção e sobre as dificuldades encontradas no Teste de Atenção Concentrada. A primeira questão foi: "Como você se sente nesse momento?" (nada alerta, não muito alerta, alerta, muito alerta). A segunda questão ("O que você achou difícil no teste?") era composta de cinco subitens, "tempo muito curto", "não lembrar os modelos", "ter que mudar a atenção constantemente entre os modelos e os desenhos a serem assinalados", "manter-se concentrado no teste" e "ter que verificar os desenhos um por um em uma sequeência definida". Era possível assinalar mais de uma alternativa e, em todos os subitens, havia a possibilidade de resposta tipo likert (concordo, concordo parcialmente e discordo), mas com possibilidade de comentário da resposta tipo aberto ("Gostaria de comentar algo sobre o teste ou alguma dificuldade que não foi apontado?").

\section{Avaliação de desempenho escolar}

Foi calculada a nota de todas as disciplinas do terceiro bimestre. Como na escola na em que a pesquisa foi realizada possui um sistema de conceitos, estes foram convertidos em números para o cálculo das médias de desempenho, conforme a seguinte regra: I (Insuficiente) $=0, S$ (Suficiente) $=$ I e PS (Plenamente Suficiente) $=2$.

\section{Procedimento}

Após coletadas as autorizações para a participação na pesquisa, ocorreu a aplicação dos instrumentos, realizada em três salas de aula, com duração de 50 minutos cada, sendo que os estudantes ficaram sentados em carteiras individuais. Os testes foram aplicados de maneira seqüencial, acompanhados pelo experimentador que forneceu instruções e esclarecimentos quanto à sua realização, na seguinte ordem: Ficha para obtenção dos dados pessoais (sendo que as perguntas sobre a dificuldade de resolução do teste de atenção deveriam ser respondidas após a execução do teste); Teste de Atenção Concentrada e Inventário de Depressão de Beck.

\section{Resultados}

Para a análise dos dados foram utilizadas estatísticas descritiva e inferencial por intermédio do Programa SPSS (Statistical Package for Social Sciences) versão II,5. Em termos de estatística inferencial foram utilizadas a prova de Spearman para a correlação e a prova de Mann-Whitney para a comparação entre os grupos com e sem sintomatologia depressiva, usando o nível de significância de $5 \%$.

A análise de freqüência das respostas da escala subjetiva de alerta indicou que os participantes se consideraram "alerta" $(56,25 \%)$ na realização dos testes, seguido por $42,19 \%$ que assinalaram "não muito alerta" e I participante (I,56\%) que assinalou o item "muito alerta". A análise de freqüência das respostas dos participantes à segunda questão da escala, "O que você achou difícil no teste?", revelou que $53,12 \%$ dos participantes concordaram que o 
tempo foi curto demais para a realização do teste e $37,34 \%$ concordaram que ter que mudar a atenção constantemente foi um fator de dificuldade. Por outro lado, os itens apontados como mais fáceis na realização do mesmo teste foram lembrar os modelos $(59,37 \%$ dos participantes) e manter-se concentrado no teste $(48,44 \%)$. Ou seja, a maioria dos participantes afirmou ter conseguido lembrar os modelos e ter conseguido se concentrar no teste. significativas. A primeira foi entre o escore do Inventário Beck de Depressão e o percentil de atenção/qualidade, sugerindo que quanto maior a sintomatologia depressiva, mais comprometida está a qualidade da atenção no teste $\left(r_{s}=-0,266, p<0,04\right)$. A segunda correlação foi entre a sintomatologia depressiva e o desempenho $\left(r_{s}=-0,257, p<0,04\right)$, indicando que quanto maior a sintomatologia de depressão, menor o desempenho escolar.

Tabela I. Freqüência e porcentagem das classificações do Inventário Beck de Depressão para cada gênero

\begin{tabular}{lrrrr}
\hline \multirow{2}{*}{ Classificação } & \multicolumn{2}{c}{ Masculino } & \multicolumn{2}{c}{ Feminino } \\
\cline { 2 - 5 } & \multicolumn{1}{c}{$\mathrm{n}$} & \multicolumn{1}{c}{$\%$} & $\mathrm{n}$ & $\%$ \\
\hline Mínimo & 20 & 76,9 & 21 & 58,3 \\
Leve & 5 & 19,2 & 13 & 36,1 \\
Moderado & 0 & 0,0 & 2 & 3,1 \\
Grave & 1 & 3,8 & 0 & 0,0 \\
\hline Total & 26 & 100,0 & 38 & 100,0 \\
\hline
\end{tabular}

O escore médio geral dos participantes no BDI foi de 8,8 pontos, com desvio-padrão de 7,6 pontos, escore mínimo de 0 e máximo de 46 pontos. A Tabela I sumaria a distribuição das freqüências por gênero.

No que se refere à prevalência de sintomas depressivos em todo o grupo, foi encontrada uma taxa de $33,8 \%(\mathrm{~N}=2 \mathrm{I})$ de indivíduos nos níveis leve a grave. Dessa taxa, I5,8\% (N=18) apresentaram uma classificação leve; I,8\% (N=2), moderado; e 0,9\% $(N=I)$, grave. Se considerados somente os níveis moderado e grave tem-se uma taxa de $4,8 \%$ de sintomas depressivos, o que corresponde a três indivíduos do total, sendo dois do sexo feminino com nível moderado e um do sexo masculino com nível severo.

Um sintoma que merece consideração particular é a ideação suicida. $\mathrm{Na}$ amostra, seis participantes $(9,7$ \%) assinalaram que têm "idéias de se matar, mas não as executaria", caracterizando um tipo de ideação passiva e dois participantes $(3,3 \%)$ assinalaram "eu me mataria se tivesse oportunidade".

Foram conduzidas análises de correlações entre os instrumentos, tendo havido duas correlações
Em relação às comparações, quando aplicado o teste de Mann-Whitney nos escores brutos do BDI, tendo como fator o gênero, foi observada diferença significativa $(U=3 \mid 8,0, p<=0,03)$, sendo que os escores dos participantes do sexo feminino foram superiores aos dos sexo masculino. Com relação ao desempenho escolar, observouse também diferença marginalmente significante entre o grupo com sintomatologia depressiva e o grupo sem sintomatologia, sendo que a média do primeiro grupo foi de 25,45 e a do segundo grupo foi de 34,60 (U=303,5, $p<0,05)$.

Finalmente, observou-se diferença entre os grupos com e sem sintomatologia de depressão quando a variável avaliada foi a qualidade da atenção (percentil). Esse resultado demonstrou que o grupo com sintomatologia depressiva cometeu mais erros ou omissões $(M=25,50)$ quando comparado ao grupo sem sintomatologia $(M=35,57)$ durante a execução do teste $(U=304,5, p<0,06)$. 


\section{Discussão}

Conforme exposto anteriormente, a prevalência de sintomatologia depressiva pode variar de forma brusca, dependendo da população avaliada, do instrumento e da metodologia usada. No presente estudo $33,8 \%$ dos participantes apresentaram sintomatologia depressiva, conforme avaliada pelo BDI. Essa incidência é alta quando comparada a dados já revisados da literatura, tais como a incidência de $22 \%$ entre respondentes de 7 a 17 anos obtida por Barbosa, Dias, Gaião e Lourenzo (1996), usando o CDI; a incidência de $20,3 \%$ entre alunos de 0 a $I 7$ anos obtida por Bahls (2002b), também usando o CDI; e a incidência de $13 \%$ entre universitários obtida por Goreinstein e Andrade (1998), usado o BDI. A prevalência maior de sintomas em indivíduos do gênero feminino observada no presente estudo também corrobora a literatura nacional e internacional (Bahls, 2002b; Baptista \& Assumpção Junior, 1999; Cruvinel, 2003; Kaltiala-Heino \& cols., 200I; Kornstein, 1997; Soares, Almeida-Filho, Botega, Coutinho \& Mari, 1994).

As evidências da relação entre sintomatologia depressiva e qualidade da atenção também era esperada, visto que diversos estudos têm revelado desempenho rebaixado de indivíduos com depressão em testes de atenção (Egeland \& cols., 2003; Rozenthal, Laks \& Engelhardt, 2004; Sévigny, Everett \& Grondin, 2003; Thomas, Goudemand \& Rousseaux, 1998). Tais relações provavelmente são devidas a diferentes padrões cognitivos de processamento de estímulos em transtornos de humor e ansiedade. De fato, indivíduos depressivos tendem a ser seletivamente mais atentos para palavras emocionalmente relevantes (Mathews, Ridgeway \& Williamson, 1996), o que poderia explicar os rebaixamentos em testes de atenção com conteúdo emocionalmente neutro.

Dessa forma, apesar da literatura ainda apresentar alguns resultados contraditórios a respeito da presença do déficit atencional em indivíduos depressivos (Lautenbacher, Spernal \& Christiankrieg, 2002; Ottowitz \& cols, 2002), o presente estudo contribuiu para corroborar a presença desse déficit. Foi demonstrando, nessa pesquisa, a relação entre depressão e atenção, tanto pela correlação significativa entre depressão e rebaixamento atencional, quanto pela diferença entre indivíduos com e sem sintomas depressivos no número de erros no teste de atenção. Tais resultados estão de acordo com a posição de Thomas e cols. (1998), segundo os quais as medidas neuropsicológicas, como as decorrentes de testes de atenção, podem ter utilidade clínica na detecção precoce de sintomas depressivos.

Algumas limitações do presente estudo devem ser levadas em consideração quando comparadas com a amostra de outros estudos. O número de participantes no presente trabalho foi humilde e a aplicação dos testes foi realizada em uma única escola, devendo-se ter muito cuidado na generalização dos resultados. De acordo com Baptista e Campos (no prelo), pesquisas não epidemiológicas e com amostras pequenas, apesar de demonstrarem prevalências específicas, devem ser vistas com cautela, pois podem apresentar vieses de amostra, baixa validade interna e, conseqüentemente, baixa validade externa.

Em se tratando de um estudo de aplicação coletiva e que revela informações pessoais, alguns estudantes podem ter omitido dados, assinalando as questões mais desejadas socialmente. Desse modo, a prevalência dos níveis moderado e grave poderiam ter sido ainda maiores. Apesar dessas limitações, a taxa encontrada indica a presença de um número significativo de adolescentes com sintomas depressivos clinicamente importantes na amostra estudada.

Uma outra questão a ser abordada refere-se à utilização dos conceitos pela escola como medida do desempenho, o que pode ter mascarado os resultados, impedindo que as diferenças entre os participantes fossem mais refinadas. Apesar de terem sido colhidos os conceitos de todas as disciplinas, esta medida de critério talvez não tenha sido suficiente para demonstrar correlações entre as variáveis atenção e desempenho, já que os conceitos utilizados pela escola fornecem menor possibilidade de variabilidade, importante para os estudos correlacionais. Se a escola utilizasse o sistema de notas numéricas (por exemplo, de zero a dez), os resultados provavelmente poderiam ter sido diferentes dos encontrados, por intermédio das análises efetuadas. 
Da mesma forma, deve-se atentar que não se objetivou, na presente pesquisa, realizar o diagnóstico de depressão, sendo que, provavelmente, a avaliação dos diferentes níveis de depressão em pacientes diagnosticados por avaliações padronizadas modificasse as associações e comparações encontradas neste estudo. Sendo assim, pesquisas futuras podem se valer de um diagnóstico padronizado para avaliar a relação entre depressão, atenção e desempenho escolar.

\section{Referências}

American Psychiatric Association (2000). DSM-IV-TR-Manual diagnóstico e estatístico de transtornos mentais ( $4^{\mathrm{a}}$ ed. revisada). Porto Alegre,RS: Artes Médicas.

Bahls, S. C. (2002a). Depressão, infância e adolescência: características clínicas. Jornal de Pediatria, 78, 359-366.

Bahls, S. C. (2002b). Epidemiologia de sintomas depressivos em adolescentes de uma escola pública em Curitiba, Brasil. Revista Brasileira de Psiquiatria, 24, 63-67.

Baptista, M. N., \& Assumpção Junior, F. B. (1999). Depressão na adolescência: uma visão multifatorial. São Paulo,SP: E.P.U.

Baptista, M. N., \& Campos, L. F. L. (2000). Avaliação longitudinal de sintomas de depressão e estresse em estudantes de psicologia. Boletim de Psicologia, L(I I3), 37-58.

Baptista, M. N., \& Oliveira, A. A. (2004). Sintomatologia de depressão e suporte familiar em adolescentes: um estudo de correlação. Revista Brasileira de Crescimento e Desenvolvimento Humano, 14(3), 58-67.

Baptista, M. N., Baptista, A. S. D., \& Oliveira, M. G. (2004). Depressão e gênero: por que mulheres se deprimem mais que homens? Em: M. N. Baptista (Org.), Suicídio e depressão: atualizações (pp.50-6I). Rio deJaneiro,RJ: Guanabara-Koogan.

Baptista, M. N., \& Campos, D. C. (no prelo). Metodologias de pesquisa em ciências: análise quantitativa e qualitativa. Rio de Janeiro,RJ: LTC.

Baptista, C. A., \& Golfeto, J. H. (2000). Prevalência de depressão em escolares de 7 a 14 anos. Revista de Psiquiatria Clínica, 27, 253-255.

Barbosa, G. A., Dias, M. R., Gaião, A. A., \& Lorenzo, W. C. G. (1996). Depressão infantil: um estudo de prevalência com - CDI. Infanto - Revista de Neuropsiquiatria da Infância e Adolescência, 14(3), 36-40.
Barbosa, A. J. G. (2004). Depressão na escola: um guia para educadores e profissionais da saúde. Em: M. N. Baptista (Org.), Suicídio e depressão: atualizações (pp. 144-157). Rio de Janeiro,RJ: Guanabara-Koogan.

Brandão, M. L. (1995). Psicofisiologia. São Paulo: Atheneu.

CEPA - Centro de Psicologia/Departamento de Estudos do CEPA (200I). Bateria fatorial CEPA - Teste de Aptidões Específicas. Rio de Janeiro,RJ: CEPA.

Cruvinel, M. (2003). Depressão infantil, rendimento escolar e estratégias de aprendizagem em alunos do ensino fundamental. Dissertação de Mestrado, Universidade Estadual de Campinas, Campinas, SP.

Cunha, J. A. (200I). Manual da versão em português das Escalas Beck. São Paulo,SP: Casa do Psicólogo.

Dalgalarrondo, P. (2000). A atenção e suas alterações. Em: P. Dalgalarrondo. Psicopatologia e Semiologia dos Transtornos Mentais (pp. 7I-73). Porto Alegre,RS: Artes Médicas.

Dell'aglio, D. D., \& Hutz, C. S. (2004). Depressäo e desempenho escolar em crianças e adolescentes institucionalizados. Psicologia: Reflexão e Crítica, 17, 35I-357.

Egeland, J., Rund, B. R., Sundet, K., Landro, N. I., Asbjornsen, A., Lund, A., Roness, A., Stordal, K. L., \& Hugdahl, K. (2003). Attention profile in schizophrenia compared with depression: differential effects of processing speed, selective attention and vigilance. Acta Psychiatric Scandinavian, 108(4), 276-284.

Engelhardt, E., Rozenthal, M., \& Laks, J. (1996). Neuropsicologia VIII - Atenção. Aspectos neuropsicológicos. Revista Brasileira de Neurologia, 32, 101-106.

Eysenck, M. W., \& Keane, M. T. (1994). Psicologia cognitiva: um manual introdutório. Porto Alegre, RS: Artes Médicas.

Goreinstein, C., \& Andrade, L. (1998). Inventário de Depressão de Beck: propriedades psicométricas da versão em português. Revista de Psiquiatria Clínica, 25, 245-250.

Graeff, F. G., \& Brandão, M. L. (1993). Distúrbios Afetivos. Em: F. G. Graeff \& M. L. Brandão, Neurobiologia das doenças mentais (pp. 79-108). São Paulo,SP: Lemos.

Instituto Nacional de Estudos e Pesquisas Educacionais Anísio Teixeira - INEP (2005). Inep divulga balanço geral do Enem 2005. Disponível em: http://www.inep.gov.br/ imprensa/noticias/enem/news05_20.htm.

Joca, S. R. L., Padovan, C. M., \& Guimarães, F. S. (2003). Estresse, depressão e hipocampo. Revista Brasileira de Psiquiatria, 25(2), 46-5I. 
Kaltiala-Heino, R., Rimpelä, M., Rantanen, P., \& Laippala, P. (200I). Adolescent depression: the role of discontinuities in life course and social support. Journal of Affective Disorder, 64, I55- 166.

Kornstein, S. G. (1997). Gender differences in depression: implications for treatment. Journal of Clinical Psychiatry, $58(15), 12-18$.

Lautenbacher, S., Spernal, J., \& Christiankrieg, J. (2002). Divided and selective attention in panic disorder: a comparative study of patients with disorder major depression and healthy controls. European Archives of Psychiatry and Clinical Neuroscience, 252, 210-213.

Liotti, M., \& Mayberg, H. S. (200I). The role of functional neuroimaging in the neuropsychology of depression. Journal of Clinical and Experimental Neuropsychology, 23(I), I2I-I36.

Mathews, A., Ridgeway, V., \& Williamson, D. A. (1996). Evidence for attention to threatening stimuli in depression. Behaviour Research and Therapy, 4, 695-705.

Ottowitz, W. E., Dougherty, D. D., \& Savage, C. R. (2002). The neural network basis for abnormalities of attention and executive function in major depression disorder: implications for application of the medical disease model to psychiatric disorders. Harvard Review Psychiatry, I0(8), 86-99.

Pérez, M. V., \& Urquijo, S. (200I). Depresión en adolescentes: relaciones com el desempeño académico. Revista de Psicologia Escolar e Educacional, 5, 49-58.

Rozenthal, M., Laks, J., \& Engelhardt, E. (2004). Aspectos neuropsicológicas da depressão. Revista de Psiquiatria, 26, 204-2। 2 .

Sarter, M., Givens, B., \& Bruno, J. P. (200I). The cognitive neurosciense of sustained attention: where top-down meets bottom-up. Brain Research Reviews, 35, I46-160.
Sévigny, M. C., Everett,J., \& Grondin, S. (2003). Depression, attention and time stimation. Brain and Cognition, 53, 35I-353.

Silva, F. T., \& Leite, J. R. (2000). Psysiological modifications and increase in state anxiety in volunteers submitted to the Stroop Color - Word Interference Test: a preliminary study. Psysiology \& Behaviour, 70, II 3-1 I8.

Soares, K. V., Almeida-Filho, N., Botega, N. J., Coutinho, E. S. F., \& Mari, J. J. (1994). Sintomas depressivos em Adolescentes: análise dos dados do estudo multicêntrico de morbidade psiquiátrica em áreas metropolitanas. Revista ABP-APAL, I6(I), II-I7.

Steingard, R. J. (2000). The neuroscience of depression in adolescence. Jornal of Affective Disorders, 6I, SI5-S2I.

Sternberg, R. J. (2000). Psicologia cognitiva. (M. R. B. Osório, trad.). Porto Alegre, RS: Artmed.

Terigi, F., \& Baquero, R. (1997). Repensando o fracasso escolar pela perspectiva psicoeducativa. Em: A. Abramovich e J. Moll (Orgs.), Para além do fracasso escolar. (pp. 105-124). Campinas, SP: Papirus.

Thomas, P, G., \& Rousseaux, M. (1998). Divided attention in major depression. Psyquiatry Research, 8I, 309-322.

Weller, E. B., \& Weller, R. A. (2000). Depression in adolescents: growing pains or true morbidity?. Journal of Affective Disorders, 6I, S9-SI3.

Williams, R. A., Hagerty, B. M., Cimprich, B., Therrien, B., Bay, E., \& Oe, H. (2000). Changes in directed attention and short-term memory in depression. Journal of Psychiatric Research, 34, 227-238.

Recebido em: 08/06/2005

Revisado em: 16/11/2005

Aprovado em: 20/12/2005

\section{Sobre os autores}

Makilim Nunes Baptista (makilim.baptista@saofrancisco.edu.br) é docente da graduação em Psicologia e do Programa de Pós-graduação Stricto Sensu em Psicologia da Universidade São Francisco.

Ricardo Franco de Lima (rilima@fcm.unicamp.br) é psicólogo formado pela Universidade São Francisco.

Alessandra Gotuzo Seabra Capovilla (alessandra.capovilla@saofrancisco.edu.br) é docente em Psicologia e do Programa de Pós-graduação Stricto Sensu em Psicologia da Universidade São Francisco.

Liana Lins Melo (liana.melo@saofrancisco.edu.br) é docente da Área de Ciências Biológicas e da Saúde da Universidade São Francisco.

Endereço para correspondência:

Rua Alexandre Rodrigues Barbosa, 45

|325|-900 - Itatiba - SP 